\title{
Design and Simulation of a Damper with Negative Stiffness for Vibration Mitigation from Drilling Equipment to a Semi-Submersible Platform
}

\author{
Yuan Zhang, ${ }^{1}$ Shuqing Wang $\left(\mathbb{D},{ }^{1}\right.$ Hui Fang $\mathbb{D},{ }^{1}$ Huawei Han, ${ }^{1,2}$ and Yihua $\mathrm{Xu}{ }^{1}$ \\ ${ }^{1}$ Shandong Provincial Key Laboratory of Ocean Engineering, Ocean University of China, Qingdao 266100, China \\ ${ }^{2}$ CIMC Raffles Offshore Pte. Ltd., Yantai 264000, China
}

Correspondence should be addressed to Shuqing Wang; shuqing@ouc.edu.cn and Hui Fang; fanghui@ouc.edu.cn

Received 19 January 2020; Revised 18 May 2020; Accepted 9 June 2020; Published 10 July 2020

Academic Editor: Jie Zhang

Copyright (c) 2020 Yuan Zhang et al. This is an open access article distributed under the Creative Commons Attribution License, which permits unrestricted use, distribution, and reproduction in any medium, provided the original work is properly cited.

\begin{abstract}
A negative-stiffness damper (NSD) that incorporates eccentric columns and high-stiffness springs is proposed for vibration mitigation from drilling equipment to a semi-submersible platform. Eccentric columns are considered as negative-stiffness elements, which can provide high static stiffness and achieve negative stiffness with hysteresis energy dissipation caused by buckling mode transition under dynamic conditions. With the assistance of high-stiffness springs, the damper possesses a hysteresis loop that can dissipate vibration energy and mitigate responses. Firstly, the hysteresis characteristics and negative stiffness of eccentric columns are analyzed. Then, the design principle of the NSD is derived, and several characteristics required for applications are investigated. In addition, an NSD system is designed for a target drilling mud pump based on measured data, and the mitigation performance is analyzed by comparing the vibration responses between cases of the proposed NSD system and the steel pedestal. The results demonstrate that the proposed system can effectively reduce the width of affected coverage and the magnitudes of vibration responses. The investigations performed in this paper not only provide the design principle of the new damper, but also prove the great potential of the proposed damper in vibration mitigation from drilling equipment to the hull of the platform.
\end{abstract}

\section{Introduction}

Vibrations generated by large-scale equipment on a semisubmersible platform are attracting more and more attentions because they can easily propagate through cabins and may cause harm to the health of the personnel and even cause structural damage [1]. Steel pedestals are commonly used to support large-scale equipment due to the high bearing capacity. However, the low damping of steel pedestals may lead to poor effects on vibration mitigation $[2,3]$. Therefore, a damper with both high bearing capacity and energy dissipation is required to mitigate the responses generated by large-scale equipment.

In recent years, nonlinear dampers have drawn increasing attentions since they possess several advantages compared with linear ones. Ibrahim [4] summarized the characteristics of many different types of nonlinear dampers, in which a group of dampers using buckled columns are being analyzed. When buckled columns are constrained laterally, they exhibit negative stiffness by providing a reaction force in the same direction as the displacement under compressions. Initially, buckled columns were used as auxiliary components in damper design. Liu et al. [5] and Huang et al. [6] proposed nonlinear dampers containing Euler buckled columns, which can be regarded as correctors with negative stiffness. A new damper using buckled columns with fixed ends was proposed and analyzed in [7-9]. Brennan et al. [10] and Fulcher et al. [11] proposed a tuned damper consisting of two buckled columns. The mitigating frequency range can be tuned by adjusting the buckling shape of the columns.

Afterwards, a new axial damper of flat-ended column was proposed. The flat-ended column possesses high static stiffness. When axial force of a column reaches critical load, 
the negative stiffness is generated through the buckling mode transition from buckling with fixed ends to pinned ends. During a loading-unloading process, the axial force-deformation curve of the flat-ended column shows a flag-shaped hysteresis loop, which makes the structure suitable for negative stiffness elements in the design of dampers. Kalathur et al. [12] analyzed the buckling behavior of compressed flat-end columns theoretically. Dong et al. [13-15] proposed dampers of columns. Both high damping and high stiffness are achieved by these dampers. Zhang et al. [16] proposed a convex eccentric column brace with wider applicability. Wu and Phillips [17] designed a shape memory alloy (SMA) capped column with elastic buckling mode jump (BMJ) mechanism, obtaining flag-shaped hysteretic damping without material failure. Wang et al. [18] designed an all-metal brace with hysteresis dissipation for impact protection of jacket platforms.

Although the high stiffness and high damping of eccentric columns are attractive for the vibration mitigation of large-scale equipment, a method of harmonizing the damper parameters with the specific equipment and dynamic loads is required before applications. In addition, the mitigation efficiency of the damper utilizing these elements in the cabin case has not been analyzed.

In this paper, a damper incorporating both eccentric columns and high-stiffness springs is proposed and investigated. The hysteresis behavior and multistage stiffness of eccentric columns are analyzed, and the design principle and dynamic analysis of the damper are clarified. Based on the design principle, a negative-stiffness damper (NSD) system is designed for a target drilling mud pump. The buckling behavior of dampers is well captured. Vibration responses of the structure within the cases of the system and the steel pedestal are compared. The results demonstrate that the negative-stiffness damper system not only greatly decreases the amplitude of vibration responses, but also effectively reduces the width of the affected coverage.

\section{Hysteresis Behavior and Multistage Stiffness of Eccentric Columns}

2.1. Numerical Simulation of the Hysteresis Behavior of an Eccentric Column. The configuration of an eccentric column is shown in Figure 1. The proposed eccentric column can be regarded as a rectangular column connected to two thick ends. A quarter of circle arc is carved for avoiding stress concentration at the junction between each end and the main column. The thickness of junction equals the difference value between $b_{e}$ and $b$. The eccentricity of an eccentric column is obtained by such difference value.

The buckling behavior of a sample column is analyzed by numerical simulation. A three-dimensional (3D) model consisting of two identical base plates and the sample column is established by utilizing the finite-element program, Abaqus 6.14. Geometric properties of the sample column are listed in Table 1. Both sizes of two base plates are $30 \mathrm{~mm} \times$ $30 \mathrm{~mm} \times 5 \mathrm{~mm}$. All parts are assigned as the material of steel (60SiMnA), and linear elastic property is applied in the simulation. The material properties are listed in Table 2.

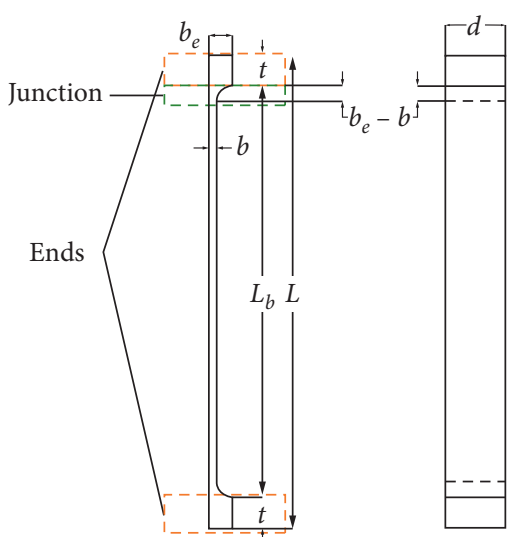

Figure 1: Configuration of an eccentric column.

TAble 1: Parameters of the sample column (unit: $\mathrm{mm}$ ).

\begin{tabular}{lccccc}
\hline$L$ & $L_{b}$ & $t$ & $b$ & $d$ & $b_{e}$ \\
\hline 400 & 360 & 18 & 3 & 15 & 5 \\
\hline
\end{tabular}

TABLE 2: Material properties.

\begin{tabular}{lcccc}
\hline Material & $\rho\left(\mathrm{kg} / \mathrm{m}^{3}\right)$ & $E(\mathrm{MPa})$ & $\mu$ & $\sigma(\mathrm{MPa})$ \\
\hline Steel $(60 \mathrm{Si} 2 \mathrm{MnA})$ & 7740 & 206000 & 0.29 & 1372 \\
\hline
\end{tabular}

All parts of the model are meshed with the element type of 8-node linear brick with reduced integration (C3D8R), and the mesh size of the column is set as $0.4 \mathrm{~mm}$ according to the convergence study. For capturing the buckling phases of the sample column, the boundary conditions of two base plates are assigned as shown in Figure 2. All contact surfaces between ends and plates are modeled as roughly contacted and two surfaces are allowed to separate. The damping of the applied material, 0.004, is transformed to the form of Rayleigh damping and applied.

Instead of a harmonic force, a harmonic displacement control is loaded on the upper base plate to avoid structural failure, which may be caused by negative stiffness. The amplitude of the harmonic displacement is $0.1 \mathrm{~mm}$ and the frequency is $1 \mathrm{~Hz}$. The implicit dynamic analysis is used in the simulation because of its advantage in dealing with nonlinear problems. The buckling mode transition process of an eccentric column in a loading-unloading cycle is summarized in Figure 3.

Buckling with fixed ends is generated when axial force reaches the first critical load, as shown in Figure 3. The boundary condition of both ends transforms from fixed to pinned mode when axial force reaches the second critical load. Then, the eccentric column goes backward to upright state directly when unloading. Time histories of axial force and deformation and the relationship between them and maximum stresses under two modes are shown in Figures 4 and 5.

The time histories of the axial force and deformation of the sample column in a cycle are merged and shown in Figure 4(a), and the relationship between axial force and deformation is shown in Figure 4(b). Six key points of axial force are marked. From point 0 to point 2, the axial force remains proportional to the axial deformation. Two jumps of 


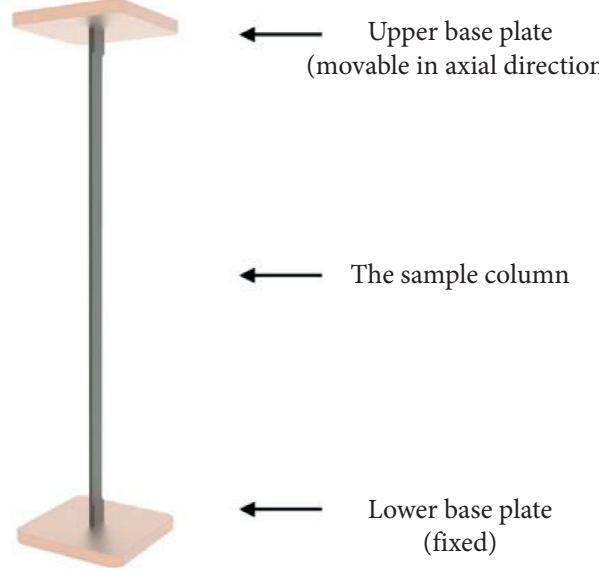

Figure 2: The 3D model of the sample column and two base plates.

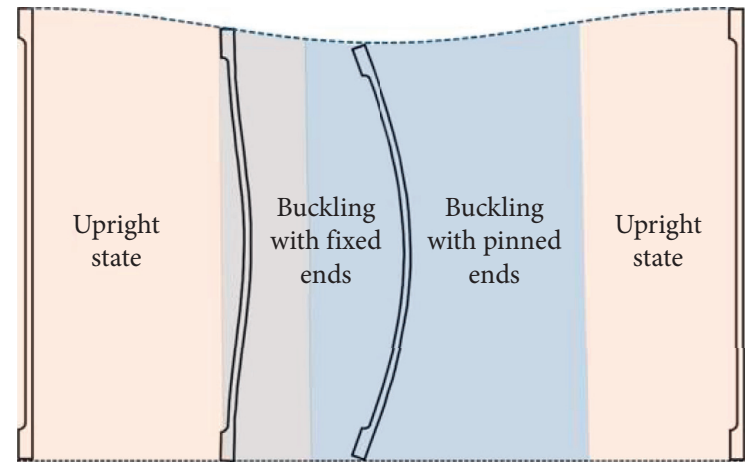

FIgURE 3: Buckling mode transition of an eccentric column in a cycle.

axial force at points 3 and 6 represent the moments when the buckling mode changes from ends fixed to pinned and then go back to upright state, respectively. It indicates that point 3 $\left(d_{3}, P_{3}\right)$ and point $6\left(d_{1}, P_{0}\right)$ can be considered as the trigger point and the attenuation point of hysteresis behavior, respectively. A D-shaped loop is formed as shown in Figure 4(b), whose area represents the energy dissipation of the column during a cycle.

Figure 5(a) represents the buckling mode with fixed ends triggered when axial force reaches the first critical load, $P_{2}$ (in Figure 4(b)). Figure 5(b) shows the buckling shape of the eccentric column when subjected to the peak displacement. The zoom-in windows clearly show the contact modes between ends and plate surfaces. Figure 5(b) shows the maximum stress in a cycle, which is far below the material yielding stress of the material.

Simulation results demonstrate that the hysteresis behavior of the sample column can be triggered in a small displacement range. The excellent bearing capacity and the large margin between the maximum stress and yielding stress proves the high applicability of the eccentric column.

2.2. Multistage Stiffness of Eccentric Columns. The multistage stiffness of eccentric columns is an external expression of the hysteresis loop, which is directly related to the instantaneous bearing capacity. Therefore, when designing an NSD, the key points associated with the loop (such as points 1, 2, 3, 4, and 6) are significant factors in evaluating the stiffness of a column at different buckling phases. The buckling phases and the multistage stiffness of the sample column are summarized and shown in Figure 6.

The hysteresis loop is divided into four phases according to the buckling mode transition- $a$ : $0-2$ (upright), $b: 2-3$ (buckling mode with fixed ends), c: 3-4-5-6-1 (buckling mode with pinned ends), and $d: 1-0$ (upright). Firstly, the stiffness value of column in phase $a$ is $21463 \mathrm{~N} / \mathrm{mm}$ and then gradually decreases to $0 \mathrm{~N} / \mathrm{mm}$ in phase $b$. Negative stiffness is triggered when the axial force reaches $P_{3}$ (in Figure 4). When the boundary condition of ends completely transforms to pinned, the axial stiffness maintains at $0 \mathrm{~N} / \mathrm{mm}$ as the axial force in this phase remains the same. Negative stiffness is also triggered at the moment of backward transition, and then the stiffness goes back to positive when the axial force returns to $P_{1}$ (in Figure 4) again.

Two buckling modes can be regarded as surface-to-surface and edge-to-surface mode, respectively. The corresponding drastic reduction in the contact area leads to a decrease in the bending stiffness of the column during loading. The restoring of bending stiffness is conducted with the increase of contact area during unloading. Two transitions in phases $b$ and $c$ represent the moments when the bending stiffness is too weak to support the compression due to the increase in deflection and when the increase of the bending stiffness is strong enough to outstrip the axial force reduction caused by the decrease in deflection, respectively. The abrupt snap effect converts the low-frequency input to a much higher-frequency one. Energy loss mechanisms are more effective at a higher frequency [13]. Therefore, with extra stiffness provided by auxiliary components, eccentric columns are able to support and mitigate a large-scale equipment.

The theoretical model summarized in [17], as listed in Table 3, can be applied to verify the simulation results and predict the stiffness of eccentric columns in damper design. Table 3 lists the axial forces and stiffness of eccentric columns in different phases.

In Table 3, $E$ is the elastic modulus, and $A$ is the area of the column section. The deflection function from point 2 to 3 is $v_{2}=\left(v_{\max } / 2\right)\left(1-\cos \left(\pi z / L_{b}\right)\right)$, and $v_{\max }=(b / 2) . z$ is the axial coordinate.

The stiffness of an eccentric column between point 0 and point $2, K_{1}$, represents the initial bearing capacity. But when slenderness ratio $\left(L_{b} / b\right)$ exceeds 100 , the value of $K_{2}$ can be regarded as $0 \mathrm{~N} / \mathrm{mm}$ because of the little difference between $P_{3}$ and $P_{2}$.

Figure 6(a) shows the theoretical and simulation results of the sample column. Although the discrepancies at point 2 and point 6 are due to the ignorance of stiffness varying in buckling mode transition processes, good agreements between two loops can still verify the simulation result.

\section{The Dynamic Characteristics of the NSD}

The dynamic characteristics of the NSD are investigated. Firstly, the design principle of the NSD is derived according 


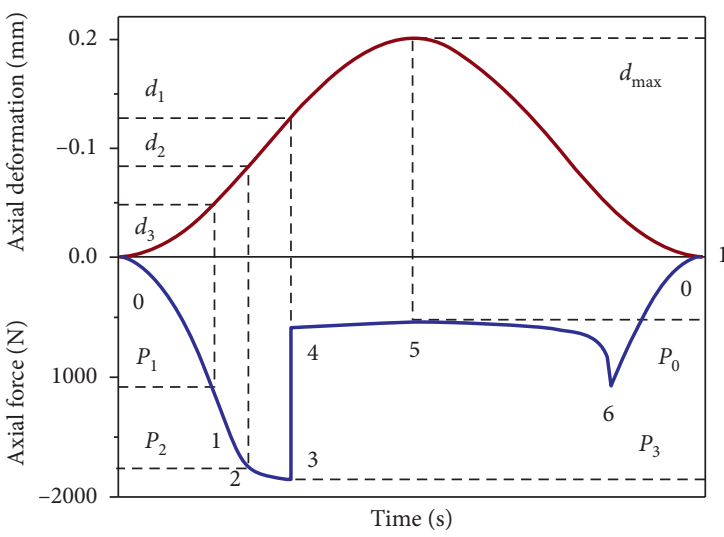

(a)

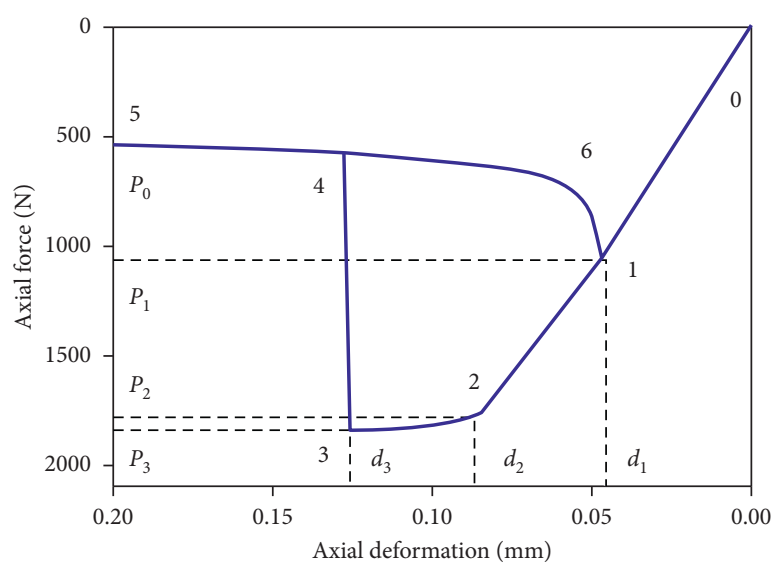

(b)

FIgURE 4: Simulation results of the sample eccentric column. (a) Time histories of force and deformation. (b) The relationship between force and deformation.

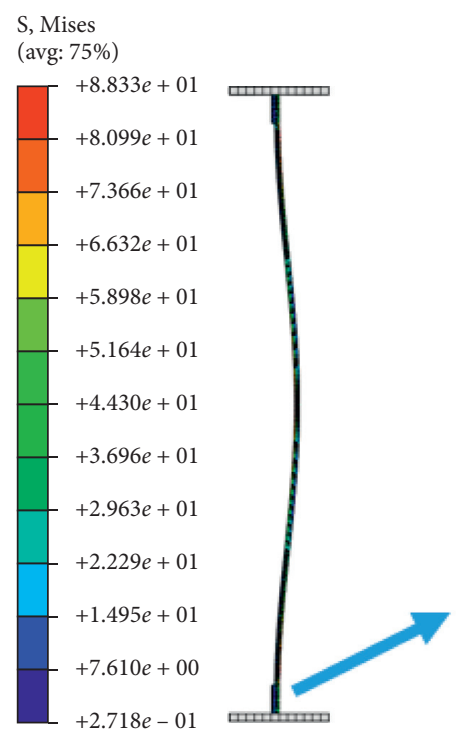

(a)

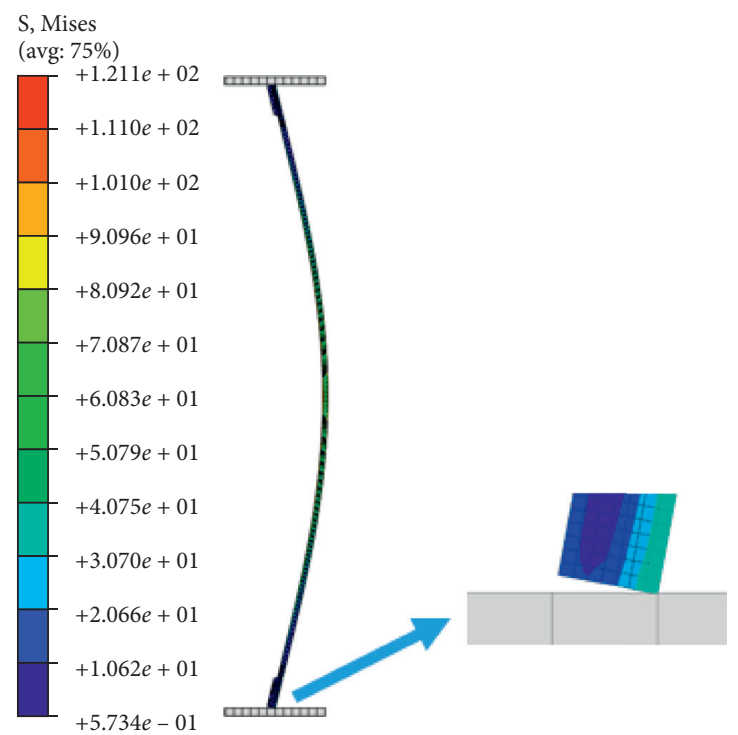

(b)

Figure 5: Maximum stresses under two buckling modes. (a) Buckling with fixed ends. (b) Buckling with pinned ends.

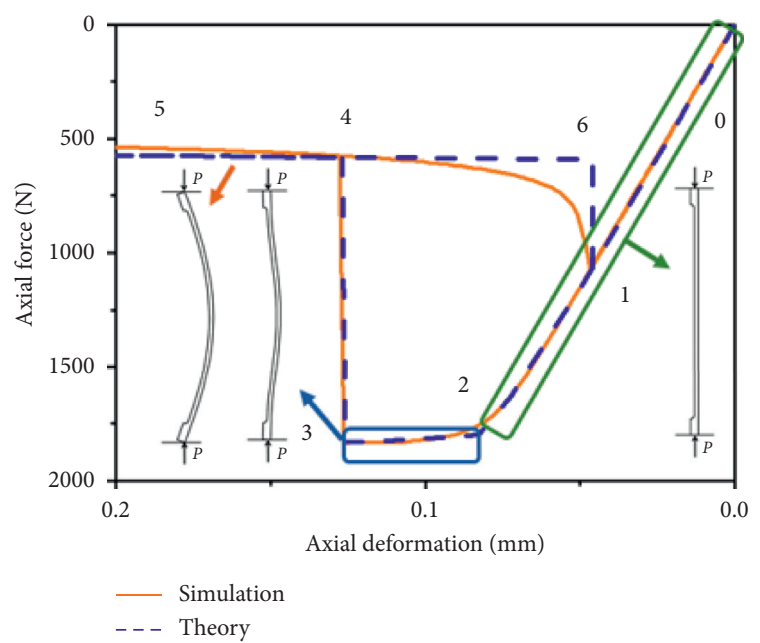

(a)

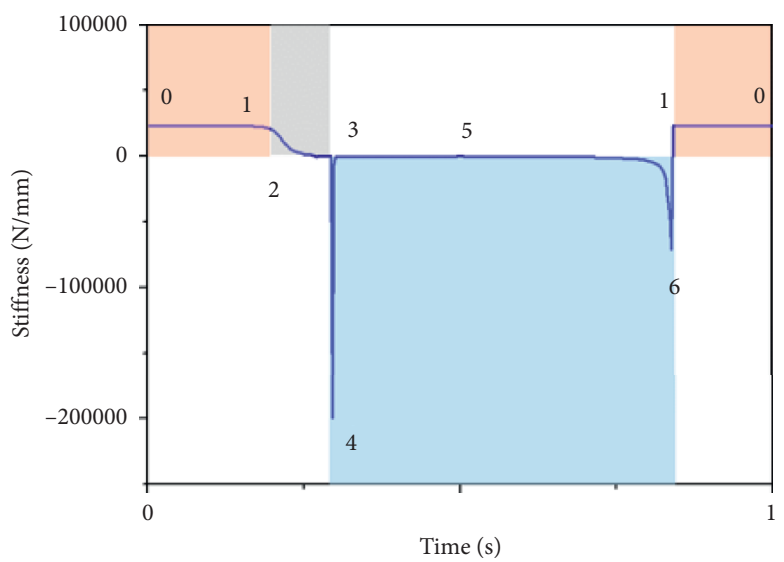

(b)

FIGURE 6: Buckling phases and the multistage stiffness of the sample column in a cycle. (a) Buckling phases. (b) The multistage stiffness. 
TABLE 3: The theoretical model of the hysteresis behavior of eccentric columns.

\begin{tabular}{lcc}
\hline Phase & Axial force & Stiffness \\
\hline $0-2$ & $P_{1}=\left(E A d / L_{b}\right)$ & $K_{1}=\left(E A / L_{b}\right)$ \\
$2-3$ & $P_{2}=\left(4 \pi^{2} E I / L_{b}^{2}\right), P_{3}=P_{2}\left(1+\left(\pi^{2} v_{\max }^{2} / 2 L_{b}^{2}\right)\right)$ & $K_{2}=\left(2 \pi^{4} E I v_{\max }^{2} / L_{b}^{4}\left(L-2 \int_{0}^{L_{b} / 2}\left(\mathrm{~d} v_{2} / \mathrm{d} z\right)^{2} \mathrm{~d} z\right)\right)$ \\
$3-4$ & $P_{4-5-6}=\left(\pi^{2} E I / L_{b}^{2}\right)$ & - \\
$4-5-6$ & - & 0 \\
$6-1$ & $P_{1}=\left(E A d / L_{b}\right)$ & $K_{1}=\left(E A / L_{b}\right)$ \\
$1-0$ & & \\
\hline
\end{tabular}

to the relationship among geometric parameters of columns, positive-stiffness elements, and exciting force. Then, the effects of several factors on hysteresis energy of the NSD and responses of the bottom structure are investigated for providing more information for applications.

\subsection{The Design Principle of the NSD. The NSD incorporates} both eccentric columns and high-stiffness springs, as shown in Figure 7. Eccentric columns can be considered as negative-stiffness elements, while high-stiffness springs can be considered as positive-stiffness elements. Several columns and springs are all arranged in the cavity of nested sleeves. In addition, ends of each column are assembled into the buckling-guiding grooves of base plates for restricting sliding, as shown in the zoom-in window of Figure 7. In this way, buckling in the specified direction is triggered when columns are compressed.

When supporting a piece of equipment, eccentric columns remain upright in equilibrium, and compression forces of both positive- and negative-stiffness parts hold the mass of equipment. When the supported equipment is running, the upper base plate starts to fluctuate up and down. Negative stiffness of each eccentric column would be triggered along with the buckling mode transition, resulting in a decrease in the axial stiffness of the NSD. The dynamic load originally carried by eccentric columns would mostly transfer to high-stiffness springs to maintain the stability of the whole damper. In the unloading process, eccentric columns return to the upright state directly, and the axial stiffness of the whole damper returns to the initial stage.

An NSD consists of several identical eccentric columns and high-stiffness springs to support large-scale equipment. The parameters and numbers of each element should be determined according to the exciting force of the target equipment. It is assumed that the mass of the supported equipment is $M$, and it generates an exciting force with the amplitude of $P_{A}$ and the rotated speed of $n$ (the unit of $n$ is $\mathrm{r} / \mathrm{s}$ ). The exciting force curve of the equipment can be written as a sinusoid:

$$
F_{E}=M g-P_{A} \sin (2 \pi n t) .
$$

The first task is to determine the number and the configuration of eccentric columns. The relationship between the geometric parameters and the exciting force can be analyzed based on the recommended locations of significant points of columns as shown in Figure 8(a).

The trigger point and attenuation point of eccentric columns should be located at the marked regions of the

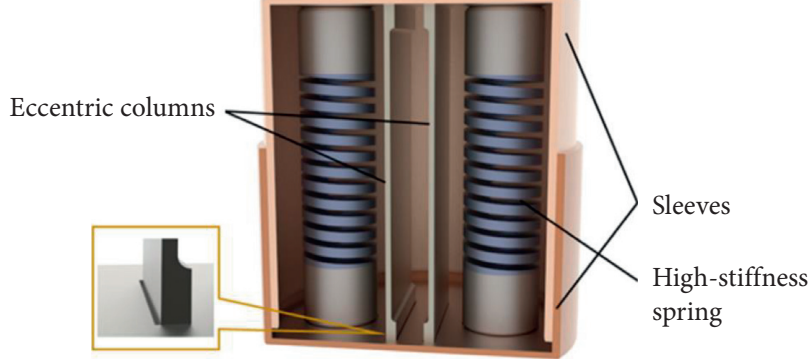

FIgURE 7: Schematic diagram of the NSD.

exciting force curve, which means the negative stiffness should be triggered before the trough and ceased before the crest. The positive- and negative-stiffness parts of the damper are composed of a number of parallel highstiffness springs (with total stiffness of $K_{S}$ ) and several identical eccentric columns, respectively. Besides, the high-stiffness springs can be precompressed by part of equipment weight $\left(M_{P}\right)$ to shorten the axial displacement of the supported equipment. To ensure the triggering of hysteresis loop, the weight of equipment should surpass the maximum bearing capacity of all columns (that is $n P_{3}<M g-M_{P} g$ ), and the minimum of dynamic load should surpass the residual bearing capacity of buckled columns (that is $n P_{4}>M g-M_{P} g-P_{A}$ ). Therefore, the relationship between geometric properties of eccentric columns and the weight of equipment can be written as follows:

$$
\begin{aligned}
\frac{4 n E \pi^{2} b^{3} d}{12 L_{b}^{2}}\left(1+\frac{\pi^{2} b^{2}}{8 L_{b}^{2}}\right) & <M g-M_{P} g \\
\frac{n \pi^{2} E b^{3} d}{12 L_{b}^{2}} & <M g-M_{P} g-P_{A} .
\end{aligned}
$$

The second task is to determine the total stiffness of all high-stiffness springs, which can be inferred from the relationship between the static equilibrium position and hysteresis loop of columns, as shown in Figure 8(b).

As high-stiffness springs are important elements to support the running equipment when negative stiffness of columns is triggered, $K_{s}$ should be determined based on $K_{1}$ and dynamic loads. Initially, the stiffness of negative stiffness part is $n K_{1}$ ( $n$ is the number of eccentric columns). Therefore, the total stiffness of the damper at the static equilibrium position, $K_{\mathrm{SEP}}$, is calculated by the sum of two parts: $n K_{1}+K_{S}$. 


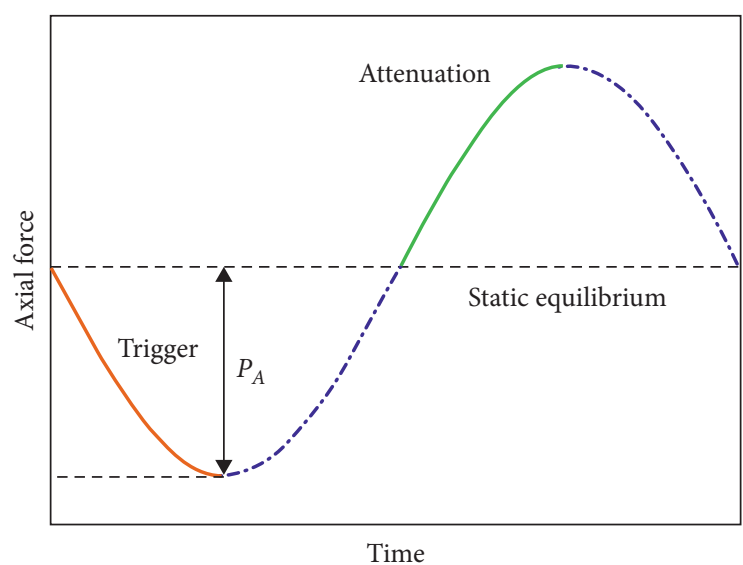

(a)

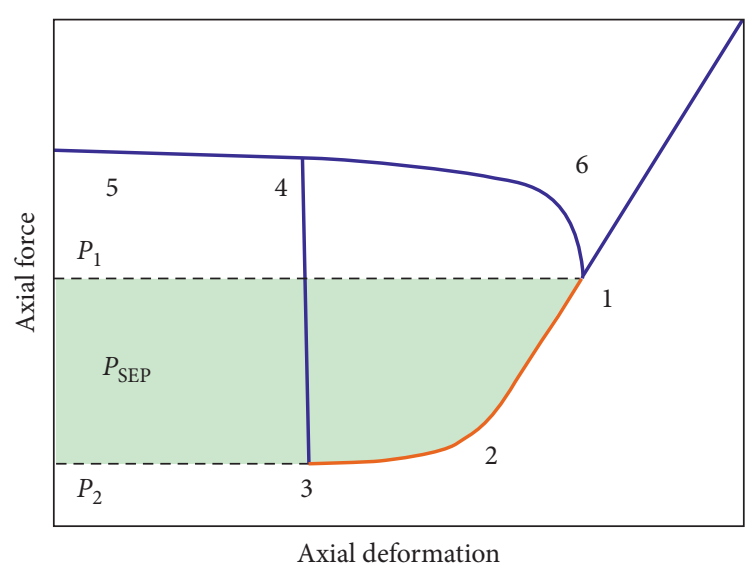

(b)

FIGURE 8: Static equilibrium position in (a) exciting force curve and (b) hysteresis loop of the column.

The axial force of a column at static equilibrium position, $P_{\mathrm{SEP}}$, is

$$
P_{\mathrm{SEP}}=\left(M-M_{P}\right) g \frac{K_{1}}{n K_{1}+K_{S}} .
$$

On the one hand, the static equilibrium position should be located between point 0 and point 3 , so the value of $P_{\text {SEP }}$ should be less than $P_{3}$. On the other hand, the range of the hysteresis loop should include the acting range of the exciting force. Therefore, total spring stiffness, axial forces, equipment weight, and exciting force amplitude should meet the following requirements:

$$
\begin{aligned}
P_{\mathrm{SEP}} & <P_{3}, \\
\left(M g-M_{P} g+P_{A}\right) \frac{K_{1}}{K_{\mathrm{SEP}}} & >P_{3} .
\end{aligned}
$$

The relationship between the value of $K_{S}$ and the column factors can be summarized as follows:

$$
\frac{K_{1}}{P_{3}}\left(M-M_{P}\right) g-n K_{1}<K_{S}<\frac{K_{1}}{P_{3}}\left(M g-M_{P} g+P_{A}\right)-n K_{1} .
$$

3.2. Hysteresis Loop of the NSD. An NSD model utilizing the sample column (described in Section 2.2) is designed according to a specific exciting force. The hysteresis loop of the whole damper and the effects of several factors on the shape of the loop are analyzed.

When the 3D column model shown in Figure 2 is subjected to an exciting force, which is written as $F_{E}=3200-1800 \sin (\pi t)$, four springs with stiffness of $4500 \mathrm{~N} / \mathrm{mm}\left(K_{S}=18000 \mathrm{~N} / \mathrm{mm}\right)$ are assigned between the upper and lower base plates according to (5). Therefore, the sample column and four springs form an NSD model. Boundary conditions remain the same as described in Section 2.2. The exciting force is loaded to the upper base plate, and implicit analysis is also adopted to capture the axial force and deformation of the NSD model. The axial force-deformation relationship of the NSD model is shown in Figure 9(a), and the time histories of axial force and deformation of each spring are integrated in Figure 9(b) due to the constant stiffness.

The clear loop demonstrates a considerable energy dissipation of the NSD model. Compared to the hysteresis loop of the single eccentric column, the loop of the NSD model can be considered as obliquely stretched due to the enlarged axial static stiffness. Initially, eccentric columns remain as the major bearing parts before the first critical load is reached. When the negative stiffness of columns is triggered at point 3 , the stiffness drop of the model causes an enlargement in axial displacement of the equipment. Then, the major bearing part converts to the springs, but eccentric columns also participate in the bearing with the residual axial force, $P_{0}$. A slight resonance process at point 4 , which cannot be analyzed by theoretical model, is motivated by buckling mode transition as shown in the dotted boxes of Figure 9(b). The results demonstrate that changing the stiffness of springs can adjust both of the deformation at static equilibrium position and maximum deformation of the damper.

The geometric parameters of the columns can affect the stiffness and the positions of key points of the NSD model [17]. Therefore, the configuration of the columns and $K_{S}$ can be adjusted to change the characteristics of the NSD model. To clarify the effects of the important parameters on the hysteresis loop of the NSD model, the hysteresis loops of NSD models with different $K_{S}$, column slenderness ratio, and depth ratio are simulated by altering one of them at a time, respectively. In addition, to figure out the rate of change in each parameter which can increase the energy dissipation under the same load, the value of $K_{S}$, column slenderness ratio, and depth ratio are changed by $1600 \mathrm{~N} /$ $\mathrm{mm}, 4$, and 0.8 twice, respectively. The hysteresis loops are shown in Figure 10.

$K_{S}$ has a little effect on the initial stiffness and energy dissipation because they are not directly involved in buckling 


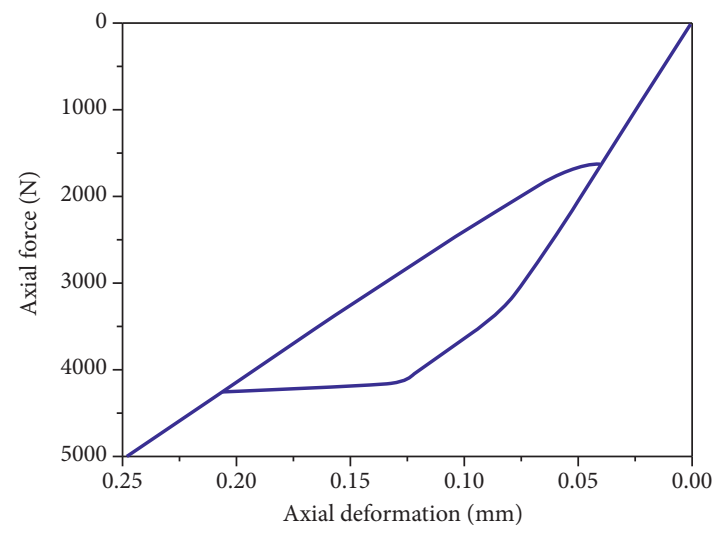

(a)

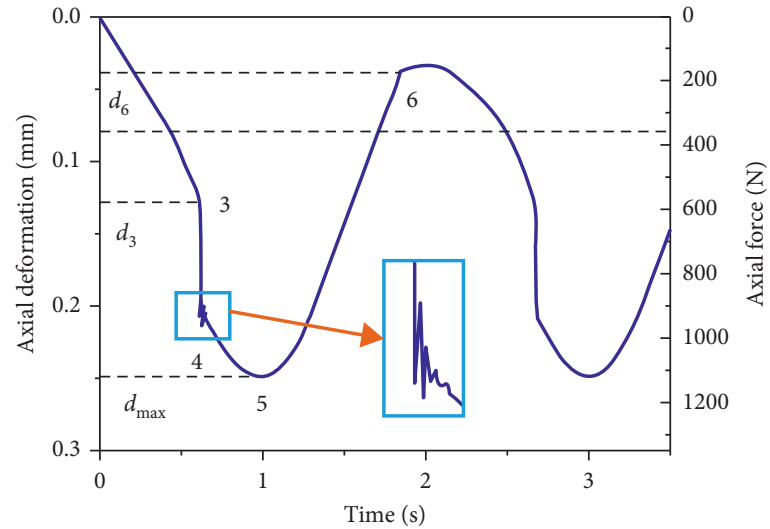

(b)

FIgURE 9: Simulation results of the NSD model. (a) Axial force-deformation relationship of the NSD model. (b) Time histories of axial deformation and force of the single spring.

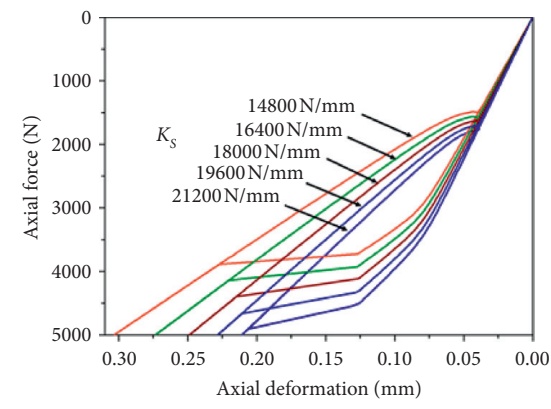

(a)

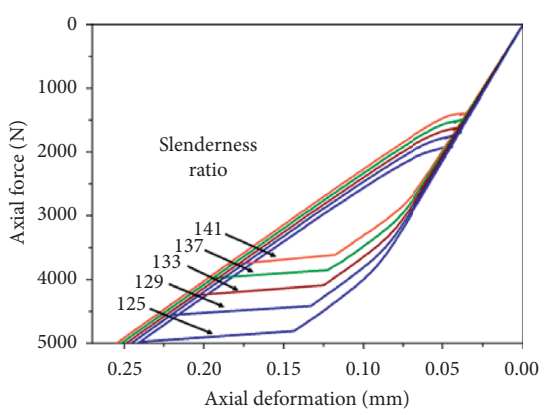

(b)

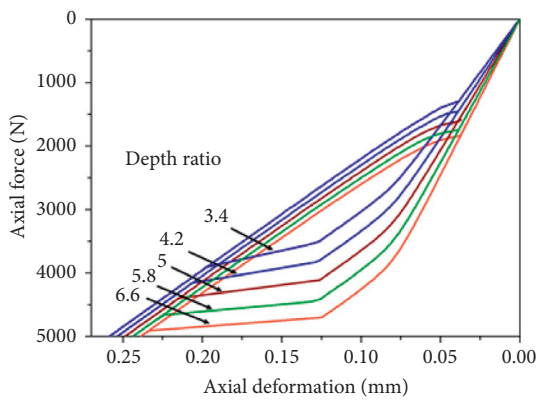

(c)

Figure 10: The effects of parameters on hysteresis loop: (a) $K_{S}$, (b) slenderness ratio, and (c) depth ratio.

hysteresis dissipation, as shown in Figure 10(a). The axial force of the trigger point is proportional to $K_{S}$, and changing $K_{S}$ can effectively adjust the maximum deformation. The slenderness ratio can significantly increase the energy dissipation, but shows little effect on the stiffness of the damper, as shown in Figure 10(b). The axial force of trigger point can be significantly increased by decreasing slenderness ratio, as $P_{3}$ of a column is proportional to the cube of $b$. In terms of depth ratio, only a great increase in depth ratio can bring about an obvious enlargement both in stiffness and in energy dissipation, as shown in Figure 10(c). Both of the stiffness and energy dissipation of an eccentric column can be intensified by decreasing slenderness ratio or increasing depth ratio. Figure 10 also demonstrates that all factors are associated with the stiffness between point 0 and point 4 , but only $K_{S}$ can affect the damper stiffness from point 5 to point 6 . Effects of the three parameters on design factors are summarized as shown in Figure 11.

The energy dissipation of the column can be enlarged by increasing the slenderness and depth ratio, as shown in Figure 11. Axial force of the trigger point can be magnified by increasing all three parameters. Axial deformation of the trigger point can only be changed by adjusting slenderness ratio. The increase in $K_{S}$ leads to a negative effect on energy dissipation, which is due to more potential energy absorbed by springs, resulting in smaller loops [18]. In general, slenderness ratio is the most significant parameter due to its strong effect on all three factors. Depth ratio follows due to its appreciable effects on energy dissipation and axial force of the trigger point. $K_{S}$ can be considered when adjusting maximum axial deformation.

3.3. The Effect of Vibration Frequency on NSD. The resonance process mentioned in Section 3.2 can be affected by vibration frequency directly, and the intensity of the resonance process may cause the distortion of hysteresis loop, leading to a loss in energy dissipation. To clarify the influence of vibration frequency on the resonance process, the NSD model described in Section 3.2 is assumed to be loaded by harmonic forces with the same amplitude of $2500 \mathrm{~N}$ and four exciting frequencies, $2.5 \mathrm{~Hz}, 5 \mathrm{~Hz}, 7.5 \mathrm{~Hz}$, and $10 \mathrm{~Hz}$. The hysteresis loops are illustrated in Figure 12.

Hysteresis behavior of the NSD model is triggered under exciting forces at all four frequencies, which basically verifies the effectiveness of this model under loads at $2.5 \sim 10 \mathrm{~Hz}$. The resonance process gets more intense and the maximum deformation develops as the vibration frequency increases. The influence of exciting frequency on displacement range of the resonance process and the 


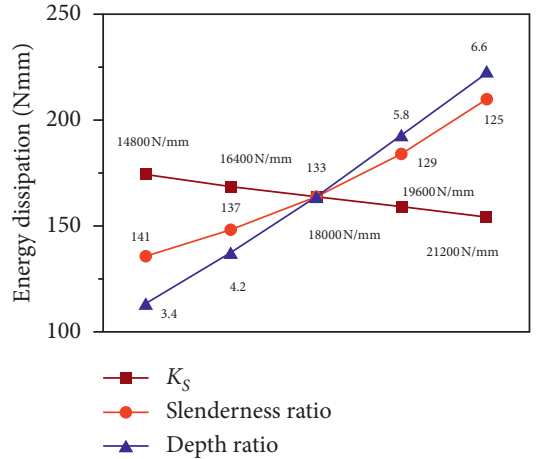

(a)

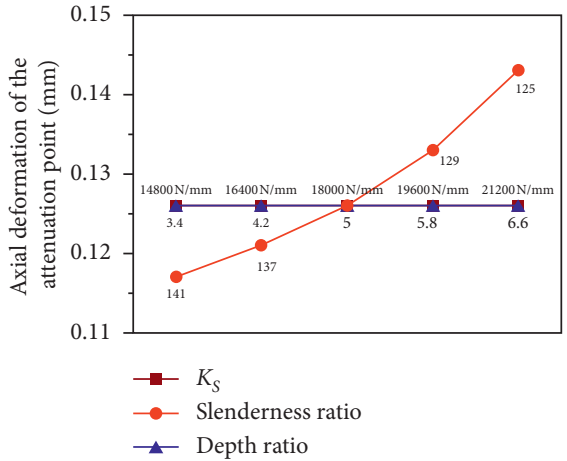

(b)

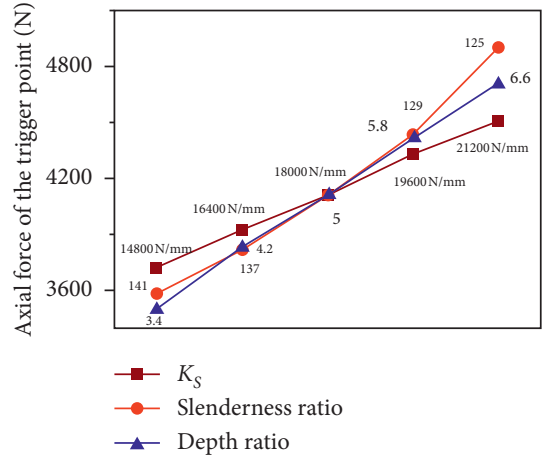

(c)

FIgURE 11: The effects of parameters on factors: (a) energy dissipation, (b) axial force, and (c) deformation of the trigger point.
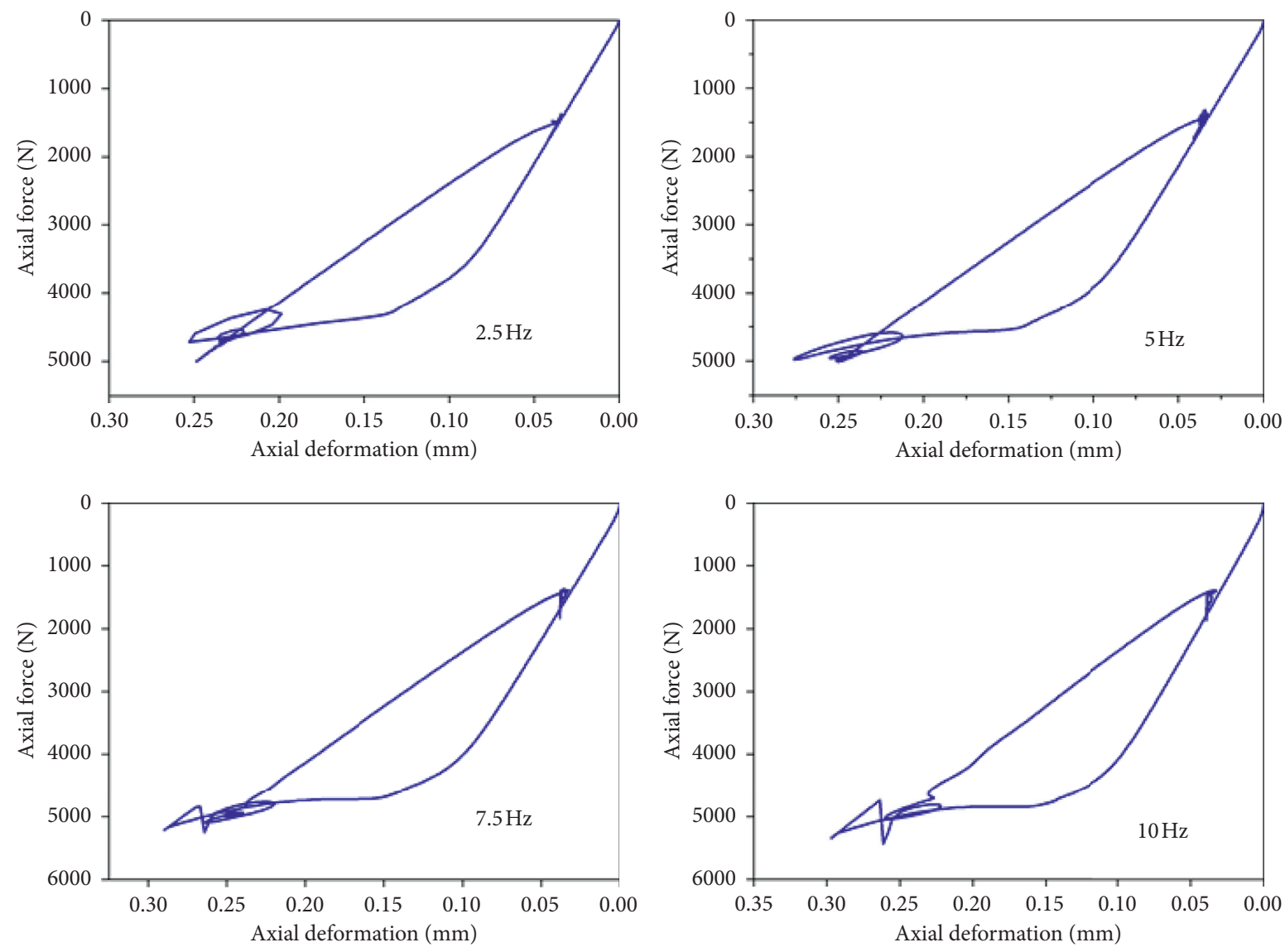

Figure 12: Hysteresis loops of the NSD model under exciting forces at $2.5 \mathrm{~Hz}, 5 \mathrm{~Hz}, 7.5 \mathrm{~Hz}$, and $10 \mathrm{~Hz}$.

maximum deformation of the NSD model are summarized as shown in Figure 13.

The displacement ranges of the resonance process and the maximum deformation of the NSD model are all increased along with the growth of exciting frequency. The histogram in Figure 13 demonstrates that the displacement range is in proportion to the vibration frequency, which means that the duration of resonance process of the column does not change along with the exciting frequency. Line chart in Figure 13 indicates that although the maximum deformation of the NSD model is enlarged, the increment gradually gets smaller. The enlargement in maximum deformation means more potential energy is absorbed. Therefore, a smaller increment in axial deformation may result in a poor performance in vibration mitigation at a higher frequency. When designing an NSD, a reasonable margin should be retained between the deformation of trigger point and maximum deformation to avoid excessive instability in practical applications. 


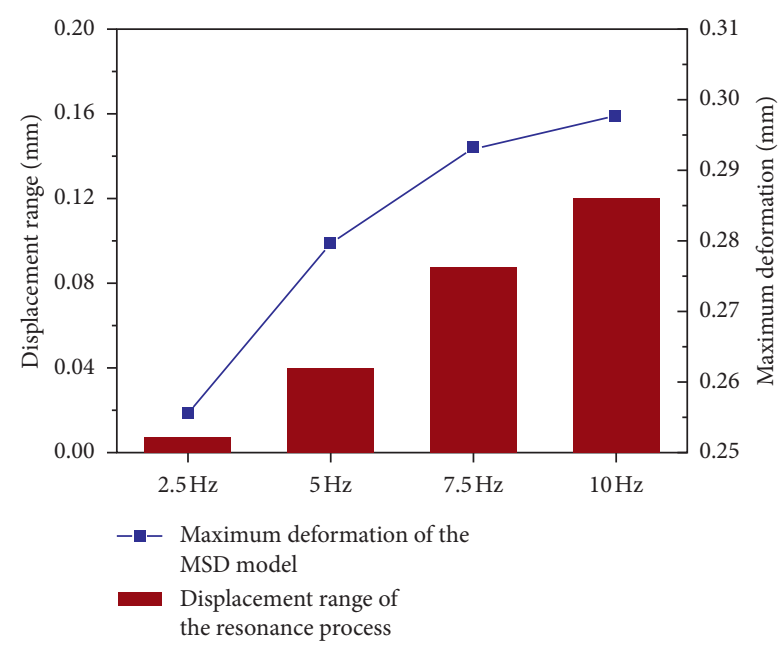

FIGURE 13: The displacement range of the resonance process and the maximum deformation of the NSD model.

3.4. The Force Transmissibility of the NSD. The force transmissibility is the basic index of mitigation performance of a damper. However, the force transmissibility of the NSD can be affected by the resonance process, which cannot be predicted by theoretical model. Therefore, numerical simulations are performed to analyze the force transmissibility of the NSD model.

To obtain the transmitted force of the NSD model, a thick bottom plate with a size of $600 \mathrm{~mm} \times 600 \mathrm{~mm} \times 9 \mathrm{~mm}$ is modeled under the lower base plate. In this case, the lower base plate is tied to the thick bottom plate, and the boundary condition of edges is pinned.

The force transmissibility $(T)$ is defined as the ratio of the amplitude of the transmitted force on bottom plate to the exciting force amplitude. The force transmissibility can be written in decibels as follows:

$$
T=20 \log _{10} \frac{f_{\mathrm{t}}}{f_{\mathrm{e}}},
$$

where $f_{\mathrm{t}}$ is the amplitude of the transmitted force, and $f_{\mathrm{e}}$ is the amplitude of exciting force.

The target mitigation frequency range of the NSD is concentrated in the range of $1 \mathrm{~Hz}$ to $10 \mathrm{~Hz}$, so only the desired frequency range $(1 \mathrm{~Hz}-10 \mathrm{~Hz})$ is discussed. Exciting force inputs at $1 \mathrm{~Hz}$ to $10 \mathrm{~Hz}$ are assigned to the center of the upper base plate, and implicit dynamic analysis is applied. The force transmissibility curves of this NSD model within force amplitudes of $2000 \mathrm{~N}, 2500 \mathrm{~N}$, and $3000 \mathrm{~N}$ and at the exciting frequency of $1 \mathrm{~Hz}$ to $10 \mathrm{~Hz}$ are obtained as shown in Figure 14.

All three curves keep horizontal at 1 to $5 \mathrm{~Hz}$ and then slightly increase at 5 to $10 \mathrm{~Hz}$, which demonstrates the stability of the NSD model in the target frequency range. The mitigation performance of the model depends on both the potential energy absorbed by column deformation and the hysteresis dissipation. However, the resonance process can affect the hysteresis loop when subjected to an exciting force at a higher frequency, and that is the reason why the transmissibility remains the same at 1 to $5 \mathrm{~Hz}$. The

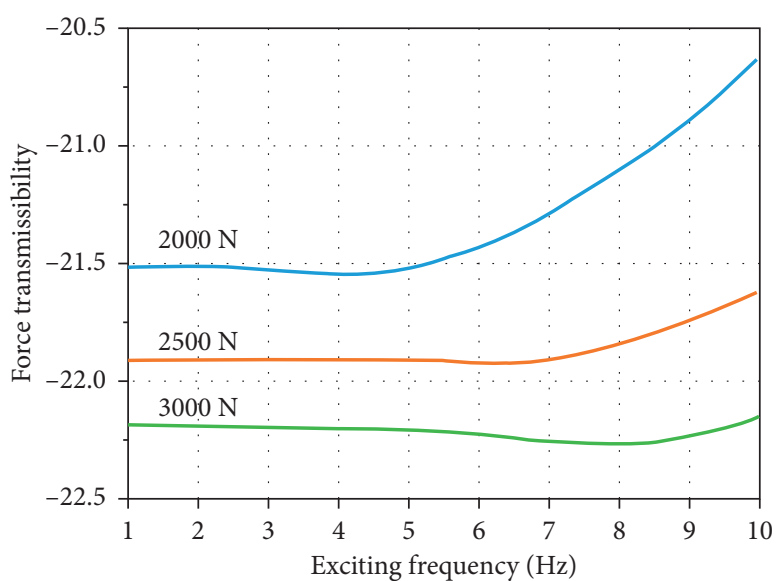

Figure 14: The effect of force amplitude on force transmissibility.

transmissibility is decreased as more energy is absorbed through larger deformation of columns. Therefore, the mitigation efficiency can be improved when subjected to a relative larger exciting force, but the increase rate is decreasing. The turning points of transmissibility curves, which represent the critical points of mitigation efficiency, move to a higher frequency when subjected to a harmonic force with larger amplitude, due to a larger margin obtained between the trigger point and the maximum deformation.

In terms of the effect of geometric parameter on force transmissibility, the slenderness ratio, which is the most significant parameter on the hysteresis behavior, is analyzed. The transmissibility curves of another two cases with slenderness ratios of 134 and 137.5, which are increased by $0.69 \%$ and $3.4 \%$, respectively, are analyzed and compared to those of the original case as shown in Figure 15.

The slightly enlarged slenderness ratio shows positive effect on the force transmissibility. The more margin retained between the trigger point and maximum deformation, the larger transmissibility and the higher frequency the turning point located. Although the energy dissipation is decreased, the augment in potential energy caused by the decreased stiffness when increasing slenderness ratio brings an improved transmissibility. However, the trend is weakening due to the nonlinearity between the slenderness ratio and $P_{3}$ of the column. It is demonstrated that pursuing energy dissipation maximization may cause a secondary problem of poor transmissibility when subjected to dynamic load at a higher frequency. Therefore, when designing an NSD, a reasonable margin between trigger point and maximum deformation should be retained according to the target frequency.

\section{Numerical Simulation of the NSD System Designed for a Drilling Mud Pump}

An NSD system is designed to mitigate the vibration from a drilling mud pump to platform structure, and its mitigation efficiency is investigated by comparing the responses of the NSD system case and the steel pedestal case. 


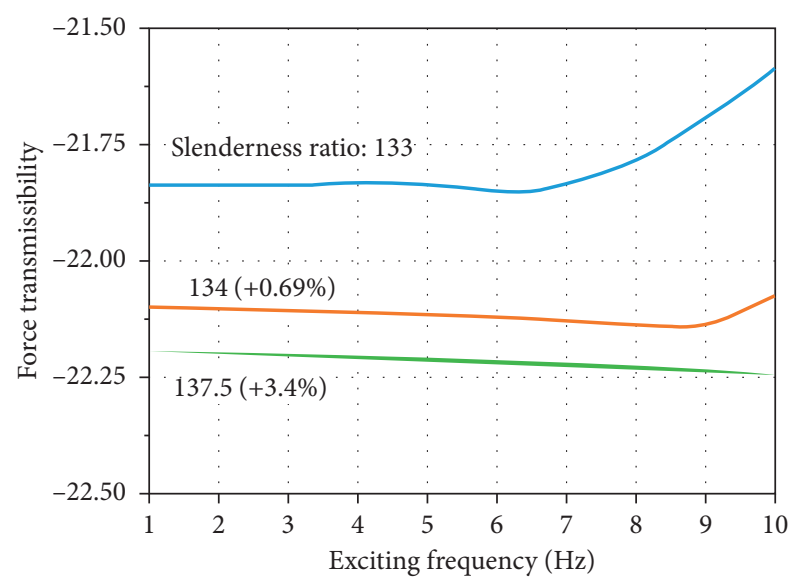

FIGURE 15: The effect of slenderness ratio on force transmissibility.

4.1. Excitation of the Drilling Mud Pump. Drilling mud pump is an important oil-mining equipment, which can deliver mud from the surface to drilling bit for cooling it and transfer the cuttings back to the surface. However, drilling mud pumps for deep-sea oil exploitation operate at low rotated speeds and they are easily to generate low-frequency exciting force with large amplitude.

The target drilling mud pump is a triplex piston pump with the size of $5543 \mathrm{~mm} \times 3290 \mathrm{~mm} \times 4528 \mathrm{~mm}$ and a weight of $39000 \mathrm{~kg}$. The drilling mud pump possesses the rotated speed of $1.75 \mathrm{r} / \mathrm{s}$. When the pump starts, a motor motivates the rotation of the pinion and then triggers the rolling of crankshaft assembly. The rolling motion transforms to the unidirectional reciprocating motion of the crossheads through the connection rods. Therefore, the exciting force of the cylinder is mainly composed of inertia forces of rolling crankshaft assembly, which consists of three components: crankshaft, pinion, and crosshead [19]. The target pump contains three cylinders, and the phase difference between each cylinder during operation is $(2 \pi / 3)$. To obtain the exciting force of the target drilling mud pump, the acceleration of the steel pedestal is measured on a semisubmersible platform as shown in Figure 16(a), and the frequency spectrum of the measured data is shown in Figure 16(b).

The measured acceleration contains several-order components in the frequency range of $0 \sim 15 \mathrm{~Hz}$, where the components at $1.75 \mathrm{~Hz}$ and $5.25 \mathrm{~Hz}$ represent the excitation loads generated by the single cylinder and the whole pump, respectively. The measured acceleration can be transformed to velocity by numerical simulation, and the exciting force of the pump can be estimated according to [20]. The exciting force under rotated speed of $1.75 \mathrm{r} / \mathrm{s}$ can be obtained as shown in Figure 17.

4.2. The Design of the NSD System. In the traditional arrangement, the drilling mud pump is placed on steel pedestals welded on the double bottom in pump cabin. The length, width, and height of the cabin are $15 \mathrm{~m}, 15 \mathrm{~m}$, and $1.8 \mathrm{~m}$, respectively. The steel pedestal consists of several webs and panels. The new proposed system consists of nine dampers in the arrangement of $3 \times 3$, as shown in Figure 18 . All dampers are fixed on the double bottom. It is assumed that all dampers are of the same configuration as they are evenly loaded.

The hysteresis behavior of dampers is assigned to be triggered and attenuated at two dotted lines as shown Figure 17, for providing the desired energy dissipation, as well as sufficient bearing capacity and margin between the trigger point and the peak. The details of the single damper are shown in Figure 19.

The NSD consists of two sleeves, eight eccentric columns, and four high-stiffness springs. To display all internal elements, the sleeves, which are utilized to restrict lateral displacement, are set as transparent. The geometric parameters of eccentric columns are shown in Table 4 . The total stiffness of springs is $380,000 \mathrm{~N} / \mathrm{mm}$, and $K_{1}$ of the column is $125,000 \mathrm{~N} / \mathrm{mm}$. For this damper, $K_{s}$ is set to be $380,000 \mathrm{~N} / \mathrm{mm}$, and the whole damper can provide the static stiffness of $1,380,000 \mathrm{~N} / \mathrm{mm}$.

The hysteresis behavior of the single NSD model is simulated on the double bottom model, and the force-deformation relationship is simulated through implicit dynamic analysis to verify the energy dissipation and the effect of resonance process of the proposed damper in a cycle. The double bottom of the pump cabin is established as shown in Figure 18. The double bottom model consists of 23307 quadrilateral elements and 9417 line elements, whose sizes are all $300 \mathrm{~mm}$. Boundary conditions of the edges are assigned as simply supported. In terms of the exciting force inputs, $33,000 \mathrm{~N}$ is preloaded to springs of the damper to shrink the axial displacement of trigger point, and the exciting force shown in Figure 17 is divided and loaded on the upper base plate of damper in the form of pressure. The force of an eccentric column and the force-deformation relationship of the damper model under a cycle are shown in Figure 20.

The hysteresis behavior of the NSD model is quite stable. The negative stiffness was not triggered at $2 \mathrm{~s}$ because the exciting force at this moment did not exceed the trigger line. Force-deformation relationship of the NSD model is shown in Figure 20(b); the maximum deformation of the upper base plate only reaches $0.327 \mathrm{~mm}$, and the maximum stress of columns is $100.9 \mathrm{MPa}$ in a cycle. The energy dissipation of an eccentric column is $418.6 \mathrm{~N} \cdot \mathrm{mm}$, and the whole dissipation of an NSD is $3348.8 \mathrm{~N} \cdot \mathrm{mm}$.

4.3. Mitigation Efficiency of the NSD System. Steel pedestals and the NSD system are modeled at the center of the cabin. The mesh size of steel pedestals is $10 \mathrm{~mm}$, and that of eccentric columns is $0.25 \mathrm{~mm}$. Exciting force is evenly assigned to the panels of two pedestals in the form of pressure. For the NSD system, each damper is assigned to the exciting force described in the last section. The total analysis time is 60 seconds, and the steady-state responses of the double bottom of the two cases are shown in Figure 21.

The results demonstrate that the NSD system can effectively decrease the maximum displacement by $75 \%$ and shrink the width of affected coverage by $46 \%$. Steel pedestals 


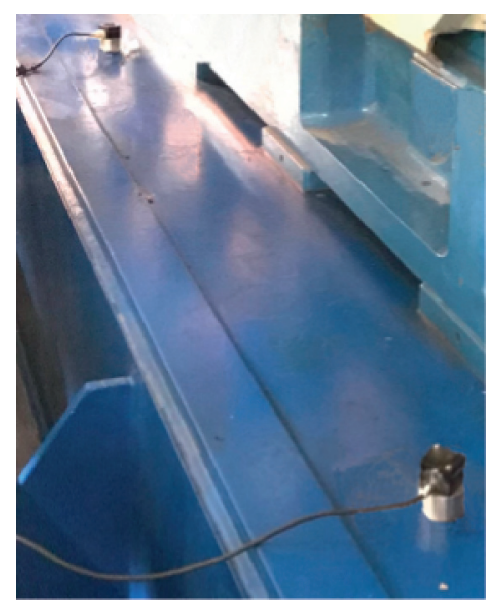

(a)

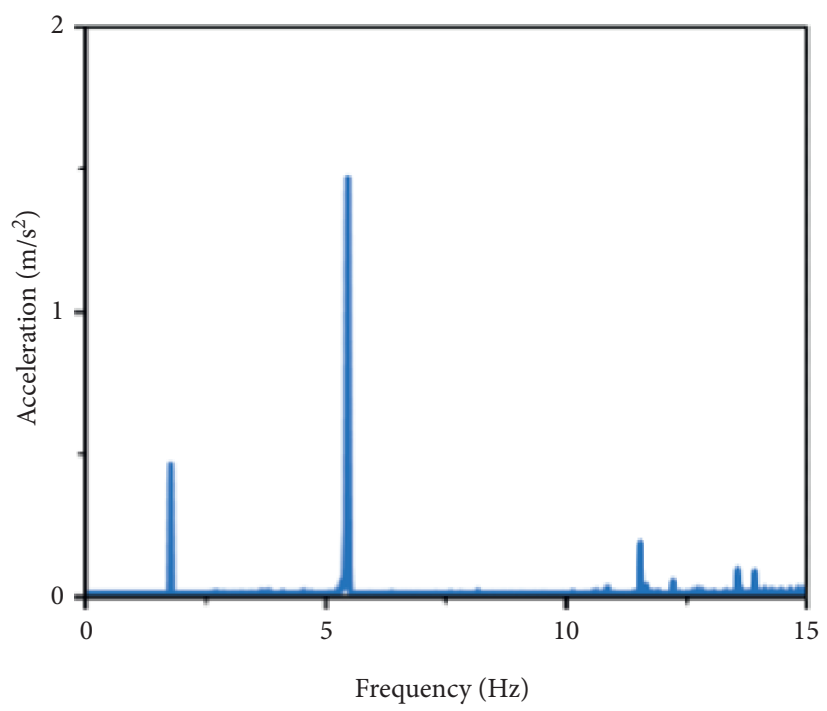

(b)

FIgURE 16: Acceleration measurement: (a) platform measurement and (b) frequency spectrum of the measured data.

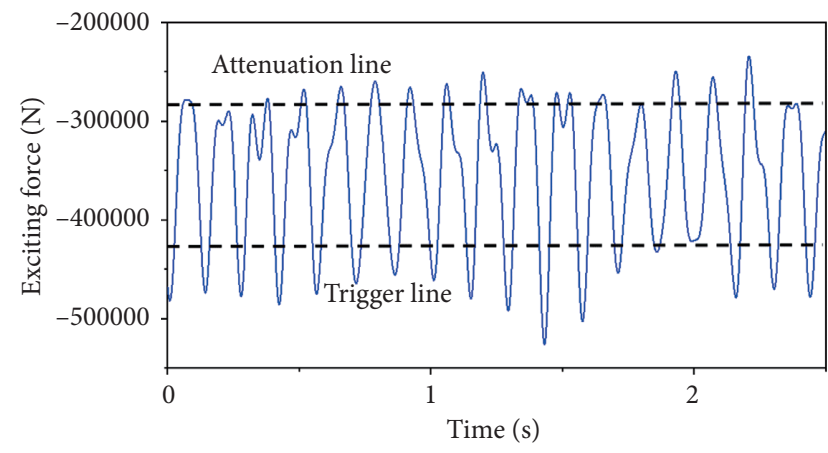

FIgURE 17: Exciting force of the drilling mud pump.

not only possess little energy dissipation, but also centralize vibration transmission, which makes it easy to induce high response over the structure.

The maximum response region is divided into two parts, which are located on the front and rear of the pump, respectively. Responses of these two regions are superimposed by displacements transmitted through surrounding dampers. On the one hand, the weight supported by the single damper is reduced. On the other hand, the deformation of columns greatly alleviates the excitation transmitted to the hull.

Responses of four test points are captured and shown in Figure 22. Time histories of four test points in the last 5 seconds are shown in Figure 23.

The displacements of static equilibrium position and amplitudes of all responses are decreased significantly, and the displacement decreases as the distance from the test point to the center of the cabin increases. Time history of steel pedestal case in Figure 23(a) represents the maximum displacement responses in the traditional arrangement. Because of the potential energy absorbed by column buckling and the hysteresis dissipation generated by buckling mode transition, the maximum displacement is decreased by $75 \%$, and the displacement amplitude is shortened by $70 \%$.

Time histories of test points 2 and 3 as shown in Figures 23(b) and 23(c) represent the maximum displacement responses in the NSD case. The equilibrium position displacement is decreased by $0.2 \mathrm{~mm}$, and the amplitude is shortened by $0.1 \mathrm{~mm}$, as shown in Figure 23(b). However, the mitigation efficiency is limited for test points 2 and 3. The mitigation efficiencies of peak displacement and response amplitude are $66 \%$ and $60 \%$, respectively.

Compared to the responses of the other three test points, response 4 in both cases is all relatively slight. The displacement of equilibrium position in the NSD case slightly decreases. The mitigation efficiencies of peak displacement and response amplitude at test point 4 are $60 \%$ and $50 \%$, respectively.

Fourier transform is conducted on four responses to analyze the frequency spectrums. Since the frequency components of all four responses in each case are basically identical, the frequency spectrum of response 1 is taken as an example, as shown in Figure 24.

The frequency spectrum in Figure 24 demonstrates that response 1 in steel pedestal case contains a tiny component of $1.75 \mathrm{~Hz}$ and a large component of $5.25 \mathrm{~Hz}$. Compared to the frequency spectrum of excitation shown in Figure 16, it is clear that the two components at $1.75 \mathrm{~Hz}$ and $5.25 \mathrm{~Hz}$ are due to the external excitation.

In terms of the response in the NSD system case, it contains a large component of $6.7 \mathrm{~Hz}$ and a smaller component of $7.3 \mathrm{~Hz}$. The $6.7 \mathrm{~Hz}$ component is induced from the NSDs, which can be proved by the frequency spectrum of the transmitted force from an eccentric column to the double bottom, as shown in Figure 25. The $7.3 \mathrm{~Hz}$ component of response 1 is superimposed by the $6.7 \mathrm{~Hz}$ components generated by the surrounding NSDs. 


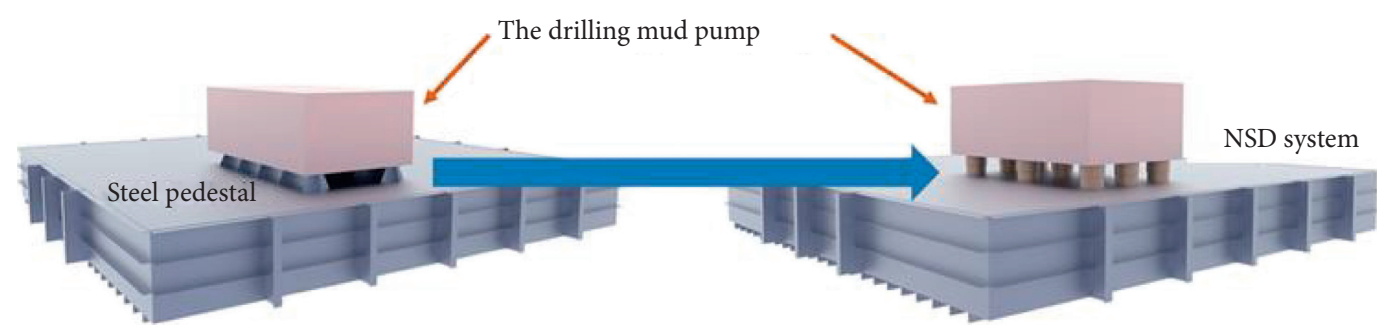

FIGURE 18: The schematic diagrams of the steel pedestal and the proposed NSD system.

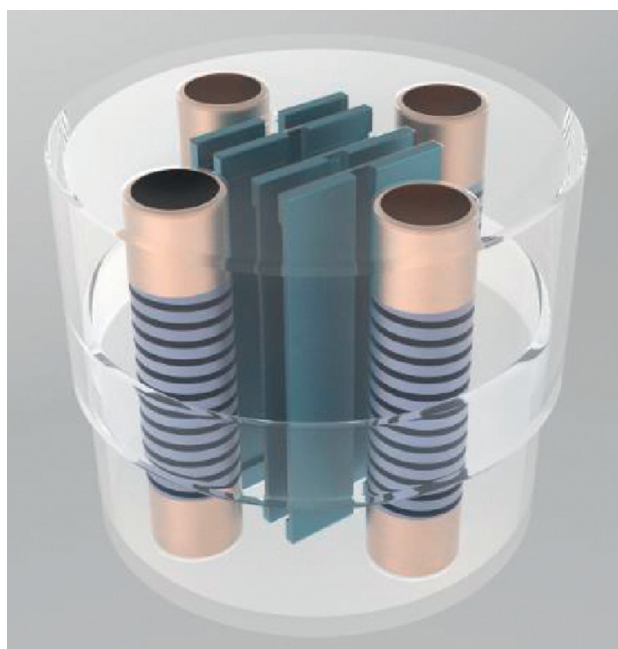

Figure 19: The schematic model of the NSD.

TABLE 4: Geometric parameters of eccentric columns (unit: mm).

\begin{tabular}{lccccc}
\hline$L$ & $L_{b}$ & $t$ & $b$ & $d$ & $b_{e}$ \\
\hline 400 & 260 & 62 & 2 & 80 & 10 \\
\hline
\end{tabular}

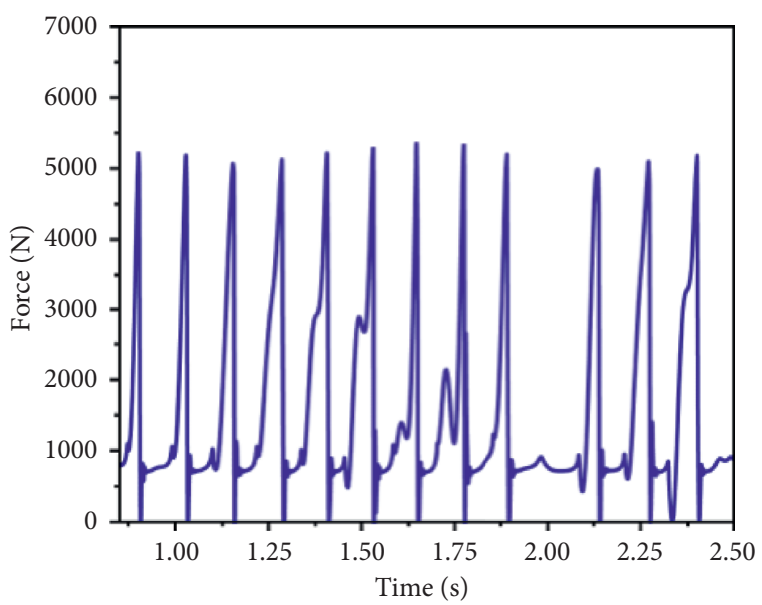

(a)

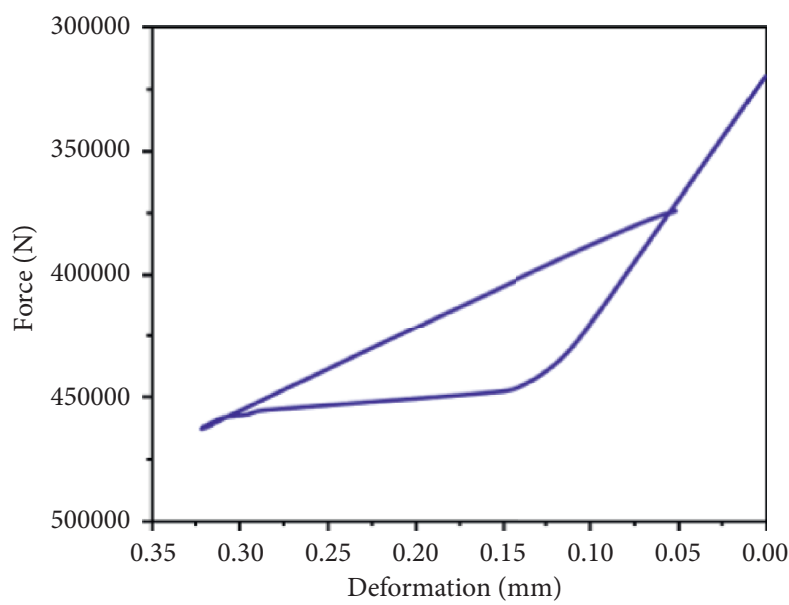

(b)

Figure 20: Simulation results of the single NSD model. (a) The time history of the force of an eccentric column. (b) Axial force-deformation relationship of the single NSD model. 


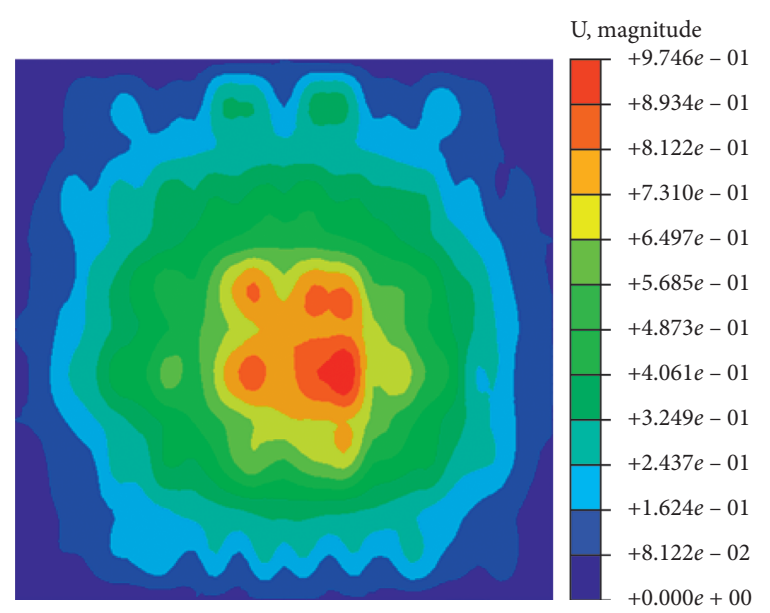

(a)

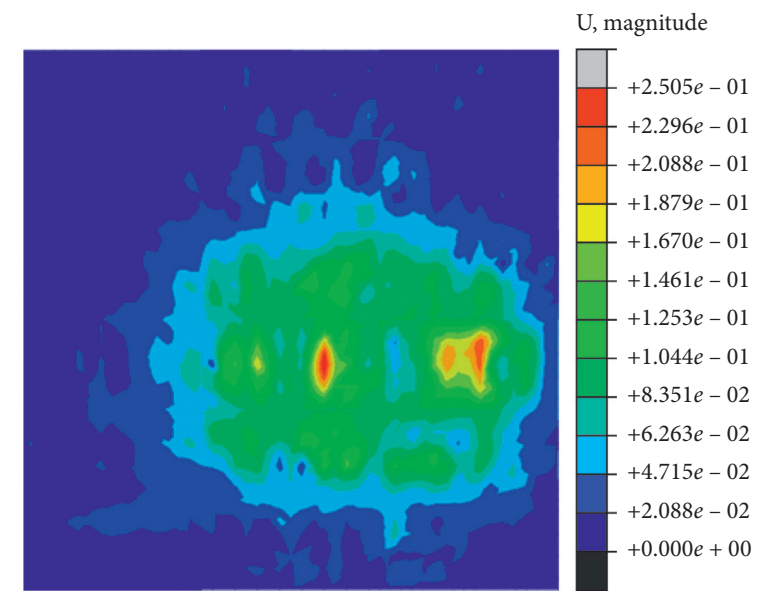

(b)

FIgURE 21: Displacement responses: (a) the steel pedestal case and (b) the NSD system case.

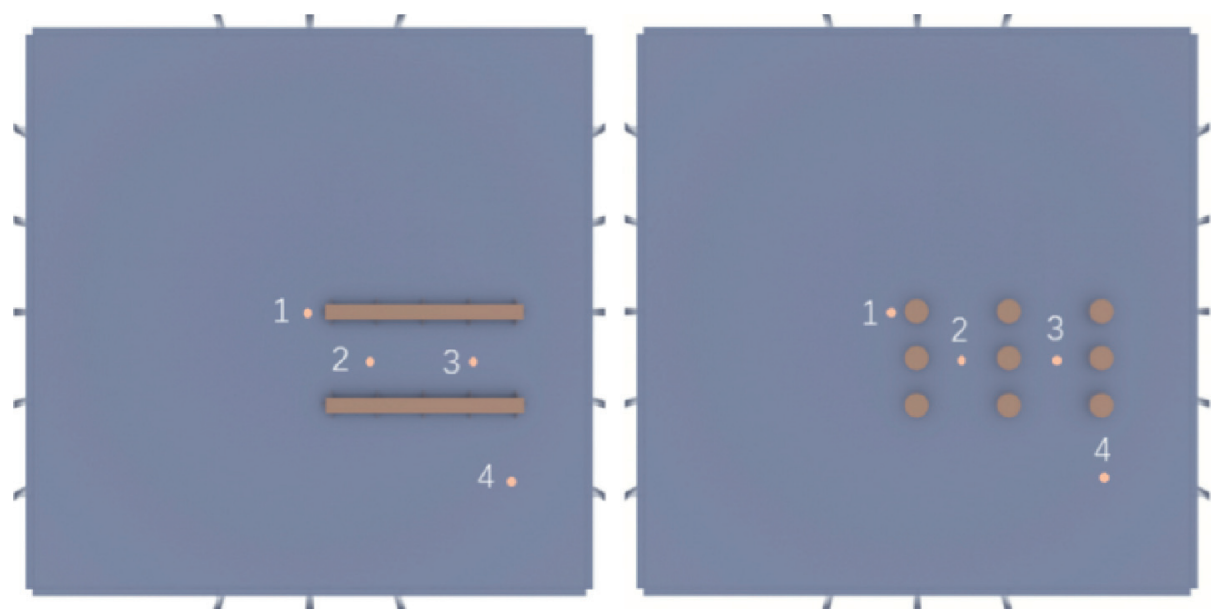

FIGURE 22: The positions of four test points on the double bottom.

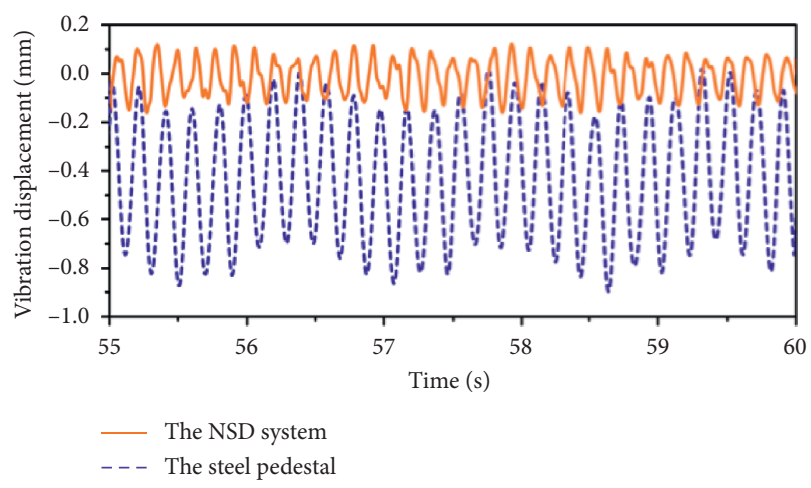

(a)

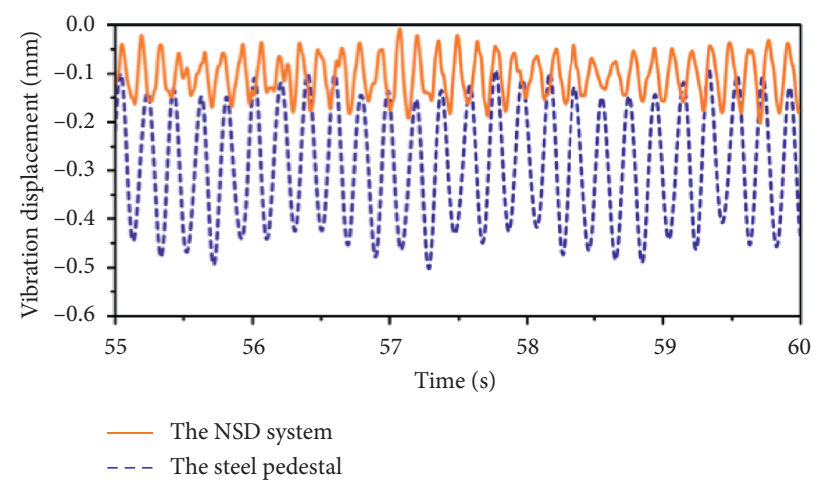

(b)

Figure 23: Continued. 


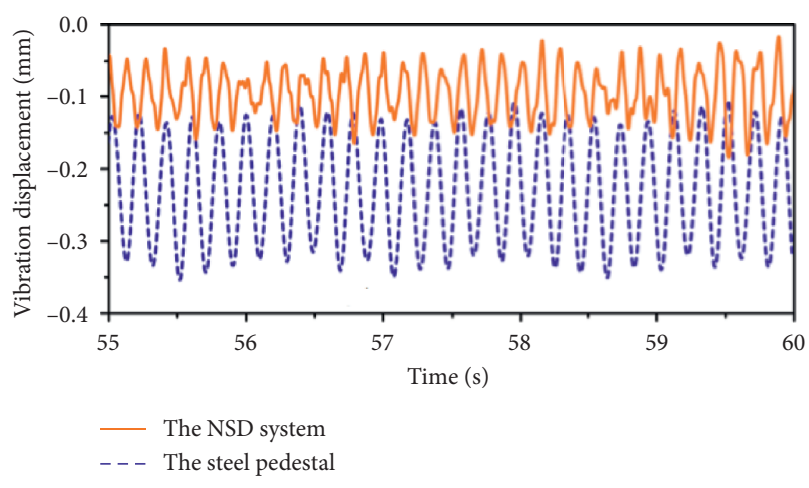

(c)

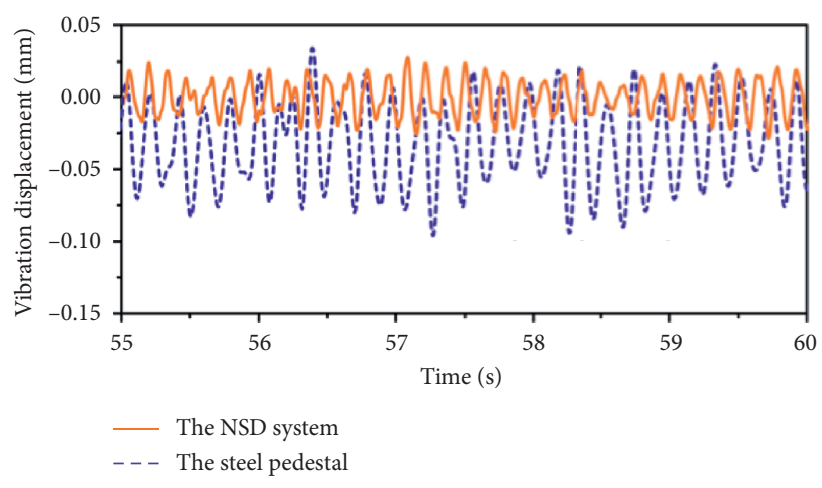

(d)

Figure 23: Time histories of four test points: (a) response 1, (b) response 2, (c) response 3, and (d) response 4.

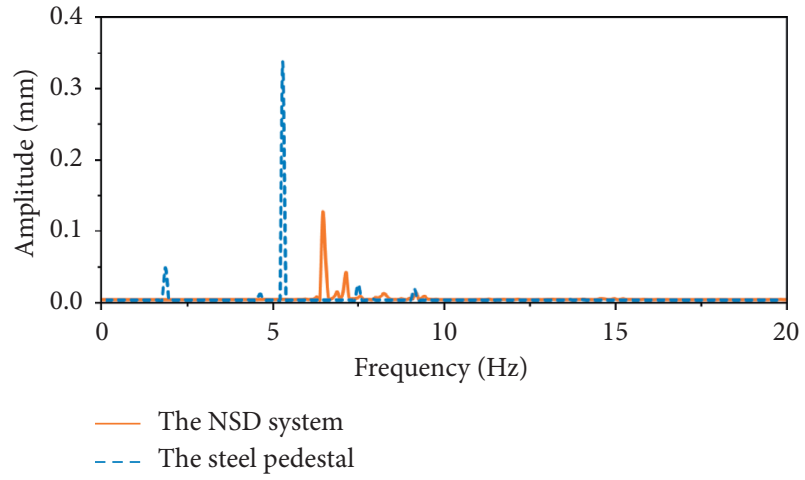

Figure 24: Frequency spectrum of response 1.

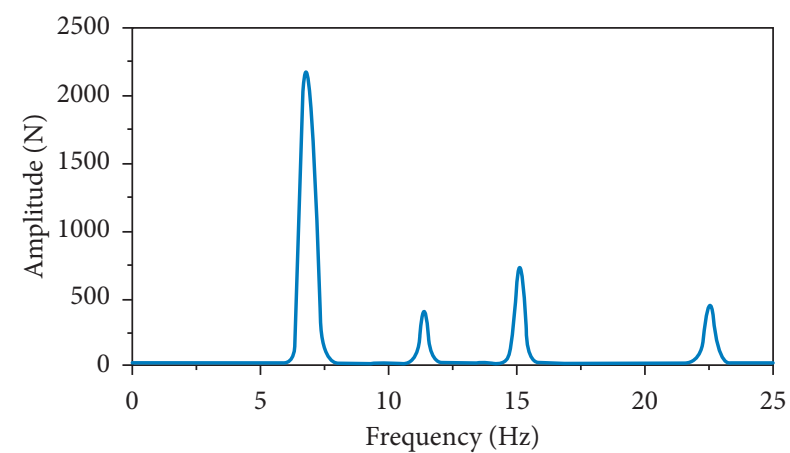

FIgURE 25: Frequency spectrum of the force of an eccentric column.

When the exciting force shown in Figure 17 is loaded on the NSDs, the exciting force can be converted to an output excitation due to the multistage stiffness of eccentric columns. And responses are induced on the double bottom of the cabin by such output excitation. The output excitation contains a large component of $6.7 \mathrm{~Hz}$, as well as three small ones at $11.4 \mathrm{~Hz}, 15.1 \mathrm{~Hz}$, and $22.5 \mathrm{~Hz}$, respectively. The component of lowest frequency of $1.75 \mathrm{~Hz}$ in the exciting force is eliminated in the output excitation due to the buckling mode transition. The $6.7 \mathrm{~Hz}$ component is converted from the $5.25 \mathrm{~Hz}$ component in exciting force. The original cycle is shortened by removing the duration of resonance process in buckling mode transition. The component of $11.4 \mathrm{~Hz}$ is generated by the corresponding component of the exciting force during phase $a$ and phase $c$ of eccentric columns, and the component of $15.1 \mathrm{~Hz}$ is generated by the buckling mode transition between phases $c$ and $d$. The component of $22.5 \mathrm{~Hz}$ represents the maximum amplitude of the resonance process. The results demonstrate that the response frequency range of the NSD model is shifted higher than that of the excitation. And the mitigation efficiency is improved because energy loss mechanisms is more effective at higher frequencies.

The response of three high-frequency components in frequency spectrum of the output excitation (as shown in Figure 25) cannot be found evidently in the frequency spectrum of response 1 (as shown in Figure 24). Because the response amplitudes transmitted to the bottom can be alleviated significantly, the mitigation efficiency of the vibration energy is improved to a higher-frequency range.

The investigation can preliminarily verify the potential utilization of the eccentric column in cabin structure, and the results also demonstrate that this NSD system can effectively mitigate the vibration responses transmitted from drilling equipment to platform structures.

\section{Conclusion}

This paper describes a kind of damper that incorporates both eccentric columns and high-stiffness springs, where the two parts are working as the positive- and negative-stiffness parts, respectively. By allowing the ends of eccentric columns to tilt, the boundary conditions of the two ends can change from fixed to pinned, which generates hysteresis damping.

The buckling of an eccentric column under harmonic displacement control is simulated. Based on the four phases distinguished by buckling modes, its hysteresis loop and multistage stiffness are theoretically analyzed. The results not only verify the accuracy of simulation, but also confirm the applicability of eccentric columns in damper design.

The design principle of an NSD is derived according to the relationship between parameters under the condition of ensuring the trigger of hysteresis behavior. The hysteresis 
loop of an NSD model under harmonic force is captured, and the influences of key factors, such as column configuration and stiffness of springs, are analyzed to provide more information for damper design.

Furthermore, an NSD system is designed for a drilling mud pump. The mitigation efficiency of the system is compared to the steel pedestal. Because of the abrupt snap effect and the hysteresis dissipation generated by the buckling mode transition, the NSD system can effectively decrease the maximum displacement by $75 \%$ and reduce the affected coverage on the structure by $46 \%$.

The investigations performed in this paper not only clarify hysteresis characteristics of the NSD, but also prove the great potential of the proposed damper in vibration mitigation of drilling equipment to platform structures.

\section{Data Availability}

The data used to support the finding of this study are available from the corresponding author upon request.

\section{Conflicts of Interest}

The authors declare that they have no conflicts of interest regarding the publication of this paper.

\section{Acknowledgments}

This research was funded by the National Science Fund for Distinguished Young Scholars (51625902), the Taishan Scholars Program of Shandong Province (TS201511016), and the "Innovation Project of 7th Generation Ultra Deep Water Drilling Platform ([2016]24)" approved by the Ministry of Industry and Information Technology of China.

\section{References}

[1] P. G. Daniel and J. J. Francisco, "Torsional system dynamics of low speed diesel engines based on instantaneous torque: application to engine diagnosis," Mechanical System and Signal Process, vol. 116, pp. 858-878, 2019.

[2] E. Diez-Jimenez, R. Rizzo, M. Gómez-García, and E. CorralAbad, "Review of passive electromagnetic devices for vibration damping and isolation," Shock and Vibration, vol. 2019, Article ID 1250707, 16 pages, 2019.

[3] E. I. Rivin, Passive Vibration Isolation, ASME Press, New York, NY, USA, 2001.

[4] R. A. Ibrahim, "Recent advances in nonlinear passive vibration isolators," Journal of Sound and Vibration, vol. 314, no. 3-5, pp. 371-452, 2008.

[5] X. Liu, X. Huang, and H. Hua, "On the characteristics of a quasi-zero stiffness isolator using Euler buckled beam as negative stiffness corrector," Journal of Sound and Vibration, vol. 332, no. 14, pp. 3359-3376, 2013.

[6] X. Huang, X. Liu, J. Sun, Z. Zhang, and H. Hua, "Vibration isolation characteristics of a nonlinear isolator using Euler buckled beam as negative stiffness corrector: a theoretical and experimental study," Journal of Sound and Vibration, vol. 333, no. 4, pp. 1132-1148, 2014.

[7] J. Z. Zhang, D. Li, M. J. Chen, and S. Dong, "An ultra-low frequency parallel connection nonlinear isolator for precision instruments," Key Engineering Materials, vol. 257-258, pp. 231-238, 2004.

[8] T. D. Le and K. K. Ahn, "Experimental investigation of a vibration isolation system using negative stiffness structure," International Journal of Mechanical Sciences, vol. 70, pp. 99-112, 2013.

[9] X. Wang, J. Zhou, D. Xu, H. Ouyang, and Y. Duan, "Force transmissibility of a two-stage vibration isolation system with quasi-zero stiffness," Nonlinear Dynamics, vol. 87, no. 1, pp. 633-646, 2017.

[10] M. J. Brennan, S. J. Elliott, and P. Bonello, "Vibration control using an adaptive tuned vibration absorber with a variable curvature stiffness element," Smart Material \& Structures, vol. 14, no. 5, pp. 1055-1065, 2005.

[11] B. A. Fulcher, D. W. Shahan, M. R. Haberman, C. C. Seepersad, and P. S. Wilson, "Analytical and experimental investigation of buckled beam as negative stiffness element for passive vibration and shock isolation systems," Journal of Vibration and Acoustics, vol. 136, Article ID 031009, 2014.

[12] H. Kalathur, T. M. Hoang, R. S. Lakes, and W. J. Drugan, "Buckling mode jump at very close load values in unattached flat-end columns: theory and experiment," Journal of Applied Mechanics, vol. 81, no. 4, Article ID 041010, 2013.

[13] L. Dong and R. Lakes, "Advanced damper with high stiffness and high hysteresis damping based on negative structural stiffness," International Journal of Solids and Structures, vol. 50, no. 14-15, pp. 2416-2423, 2013.

[14] L. Dong and R. S. Lakes, "Advanced damper with negative structural stiffness elements," Smart Material \& Structures, vol. 21, pp. 416-422, 2012.

[15] S. P. Balch and R. S. Lakes, "Lumped negative stiffness damper for absorption of flexural waves in a rod," Smart Material \& Structures, vol. 26, Article ID 045022, 2017.

[16] Y. Zhang, S. Wang, H. Fang, Z. Zhang, and X. Yuan, "Investigation of the hysteresis behavior of a convex column brace based on buckling mode transition mechanism," Advances in Mechanical Engineering, vol. 11, no. 5, pp. 1-17, 2019.

[17] J. Wu and B. M. Phillips, "Passive self-centering hysteretic damping brace based on the elastic buckling mode jump mechanism of a capped column," Engineering Structures, vol. 134, pp. 276-288, 2017.

[18] S. Wang, X. Meng, S. Ji, H. Fang, Y. Liu, and L. Duan, “Allmetal brace with hysteresis dissipation for impact protection of jacket platforms," Marine Structures, vol. 66, pp. 1-15, 2019.

[19] S. Deng, J. Pei, Y. Wang, and B. Liu, "Research on drilling mud pump fault diagnosis based on fusion of acoustic emission and vibration technology," Insight-Non-Destructive Testing and Condition Monitoring, vol. 59, no. 8, pp. 415-423, 2017.

[20] F. Pang, H. Song, X. Miu, and X. Wang, "Impedance analysis method applied in underwater acoustic radiation analysis of ship," Journal of Vibration and Shock, vol. 35, pp. 17-22, 2016, in Chinese. 